Article

\title{
Metabolic Profiling of Candida auris, a Newly-Emerging Multi-Drug Resistant Candida Species, by GC-MS
}

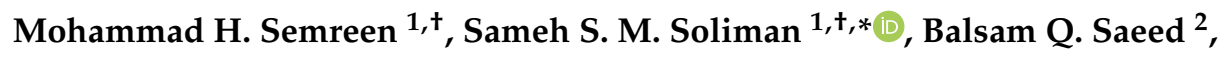 \\ Abdullah Alqarihi ${ }^{3}\left(\mathbb{D}\right.$, Priya Uppuluri ${ }^{3,4}{ }^{-}$and Ashraf S. Ibrahim ${ }^{3,4}(0)$ \\ 1 Sharjah Institute for Medical Research and College of Pharmacy, University of Sharjah, P.O. Box 27272, \\ Sharjah, UAE; msemreen@sharjah.ac.ae \\ 2 Department of Clinical Sciences, College of Medicine, University of Sharjah, P.O. Box 27272, Sharjah, UAE; \\ bsaeed@sharjah.ac.ae \\ 3 Division of Infectious Diseases, Los Angeles Biomedical Research Institute, Harbor-UCLA Medical Center, \\ Torrance, CA 90509, USA; aalqarihi@labiomed.org (A.A.); puppuluri@labiomed.org (P.U.); \\ ibrahim@labiomed.org (A.S.I.) \\ 4 David Geffen School of Medicine at UCLA, Los Angeles, CA 90095, USA \\ * Correspondence: ssoliman@sharjah.ac.ae; Tel.: 065057472 \\ + These authors have contributed to the work equally.
}

Received: 10 November 2018; Accepted: 18 January 2019; Published: 22 January 2019

check for updates

\begin{abstract}
Candida auris, a newly-emerging Candida species, is a serious global health threat due to its multi-drug resistant pattern, difficulty to diagnose, and the high mortality associated with its invasive and bloodstream infections. Unlike C. albicans, and C. dubliniensis which can form true hyphae, $C$. auris grows as yeast or pseudohyphae and is capable of developing biofilms. The reasons for the inability of $C$. auris to form true hyphae are currently unknown. Metabolites secreted by microorganisms, including Candida, are known as important factors in controlling morphogenesis and pathogenesis. Metabolic profiling of C. auris and C. albicans cultures was performed using gas chromatography-mass spectrometry (GC-MS). Compared to C. albicans, C. auris secreted several hyphae-inhibiting metabolites, including phenylethyl, benzyl and isoamyl alcohols. Furthermore, a biofilm-forming metabolite—tyrosol—was identified. On the other hand, several other biomarkers identified from $C$. auris but not from $C$. albicans cultures may be produced by the organism to overcome the host immune system or control fungal adaptations, and hence ease its invasion and infections. The results from this study are considered as the first identification of $C$. auris metabolic activities as a step forward to understand its virulence mechanisms.
\end{abstract}

Keywords: Candida auris; metabolic profiling; GC-MS; morphogenesis; hyphae; yeast

\section{Introduction}

Candida species, in particular C. albicans, are responsible for most fungal infections in humans. Although Candida species can cause superficial mucosal and skin infections, they can generate serious threats to immunocompromised patients, including fatal bloodstream candidiasis [1]. In recent years, the Centers for Disease Control and Prevention (CDC) has designated C. auris as an emerging serious global health threat mainly because of its multidrug-resistant pattern associated with high mortality rate that can reach $60 \%$ [2,3], difficulty to diagnose [4] and recent outbreaks in healthcare settings owing to the yeast persistence as a colonizer on abiotic surfaces [5].

C. auris was first identified in Japan on 2009 [6]. Since then, infections due to C. auris have been reported from over a dozen countries, including United States, Canada, Colombia, Germany, India, 
Israel, Japan, Kenya, Norway, Pakistan, Spain, South Africa, South Korea, the United Kingdom, Venezuela, Kuwait and Oman [7]. Phylogenetic analysis indicates that $C$. auris is a recent and simultaneous emergent strain in different geographical areas [8]. This yeast can be recovered from several human specimens, including sterile body fluids, ears, wounds and mucocutaneous swabs. However, the major manifestation of the infection includes invasive and bloodstream infections [9]. Like other Candida species, there is no indication that it can cause true respiratory, urinary, and skin and soft tissue infections despite being isolated from such samples [10,11]. Individuals who are at risk of acquiring $C$. auris infections include hospitalized and nursing home patients. These patients usually have comorbidities that is likely to contribute with the multi-drug resistant nature of the yeast to the lethality of the infection [12]. Importantly, C. auris is often misdiagnosed as C. haemulonii. Thus, the CDC recommends further testing whenever a diagnosis of C. haemulonii is identified [4]. As $C$. auris continues to emerge throughout the world, vital measures are required to slow down its spread and to reduce the environmental contamination in healthcare facilities and to improve diagnosis of the infection.

C. auris exhibits round-to-ovoid yeast morphology and, in some reports, ability to form pseudohyphae without the formation of true hyphae [12]. A major strategy used by Candida albicans to control its morphogenesis is the production of phenotype-switching metabolites [13]. The concentration of these metabolites increases to a critical threshold, which in turn triggers a morphogenetic regulatory response [14]. Several C. albicans metabolites, including farnesol, are known to inhibit yeast-to-hyphae formation [15]. In contrast, C. albicans tyrosol induces filamentation and hence biofilm formation [16]. Other reported hyphae-inhibiting alcohols include phenylethyl [17], isoamyl and benzyl alcohols in addition to tryptophol [18].

We conducted GC-MS analysis to compare the metabolic profile of $C$. auris to those of $C$. albicans. GC-MS is a powerful technique used to establish a comprehensive quantitative metabolic profiling of volatile small molecules from any complex biological samples [19]. The results showed that $C$. auris produces metabolites consistent with those promoting yeast formation.

\section{Results and Discussion}

C. auris or C. albicans cultures were extracted and derivatized prior to GC-MS analysis in order to enhance the appearance of metabolic spectrum and increase the detection limits of the metabolites. The identified metabolites from Candida cultures were classified in relation to their possible morphogenic and virulence activities.

\subsection{Superior Production of Hyphae-Inhibitory Metabolites by C. auris}

It is reported that, $C$. albicans responds to environmental changes by developing different polymorphic forms including yeast, pseudo-hyphae and true hyphae $[20,21]$. Phenotype-switching metabolites play a critical role in C. albicans morphogenesis [22]. Unlike C. albicans, C. auris grows readily as yeast form and does not produce true hyphae $[3,12,23]$. Further reports indicated that C. auris is capable of forming biofilms [24] and adhering to catheter material [25].

We wanted to investigate if metabolites production by $C$. auris is consistent with its yeast form, even when grown under hyphae-promoting conditions. Thus a preliminary study was conducted by growing C. auris in culture conditions that mimic the development of hyphae in C. albicans (Figure 1). Cell free supernatants from cultures of $C$. auris CAU09 and C. albicans were then collected and separately screened for the presence of similar metabolites using GC-MS analysis (Figure 2A-D). The results showed that $C$. auris CAU09 cultures secreted metabolites particularly: 1) aromatic alcohols such as phenylethyl alcohol, benzyl alcohol, isoamyl alcohol and tyrosol; and 2) acids such as benzoic, benzenacetic, glyceric and others that were not identified in C. albicans cultures (Figure 2A,B and Figure 3).

Metabolites known to be involved in Candida morphogenic changes were grouped and their average relative percentages were compared to each other in C. auris CAU09 strain using Box-and-Whiskers Plots (Figure 4A). The plots showed that there was a significant increase in the 
production of both phenylethyl alcohol $(11.39 \pm 0.5 \%)$ and tyrosol $(3.137 \pm 0.4)$. In addition, a less prominent increase in palmitelaidic acid $(1.8 \pm 0.34)$, and at lower extent, benzyl alcohol $(0.2 \pm 0.06 \%)$ as well as isoamyl alcohol $(0.57 \pm 0.19 \%)$ was detected (Figure $4 \mathrm{~A}$ and Supplementary Table S1).

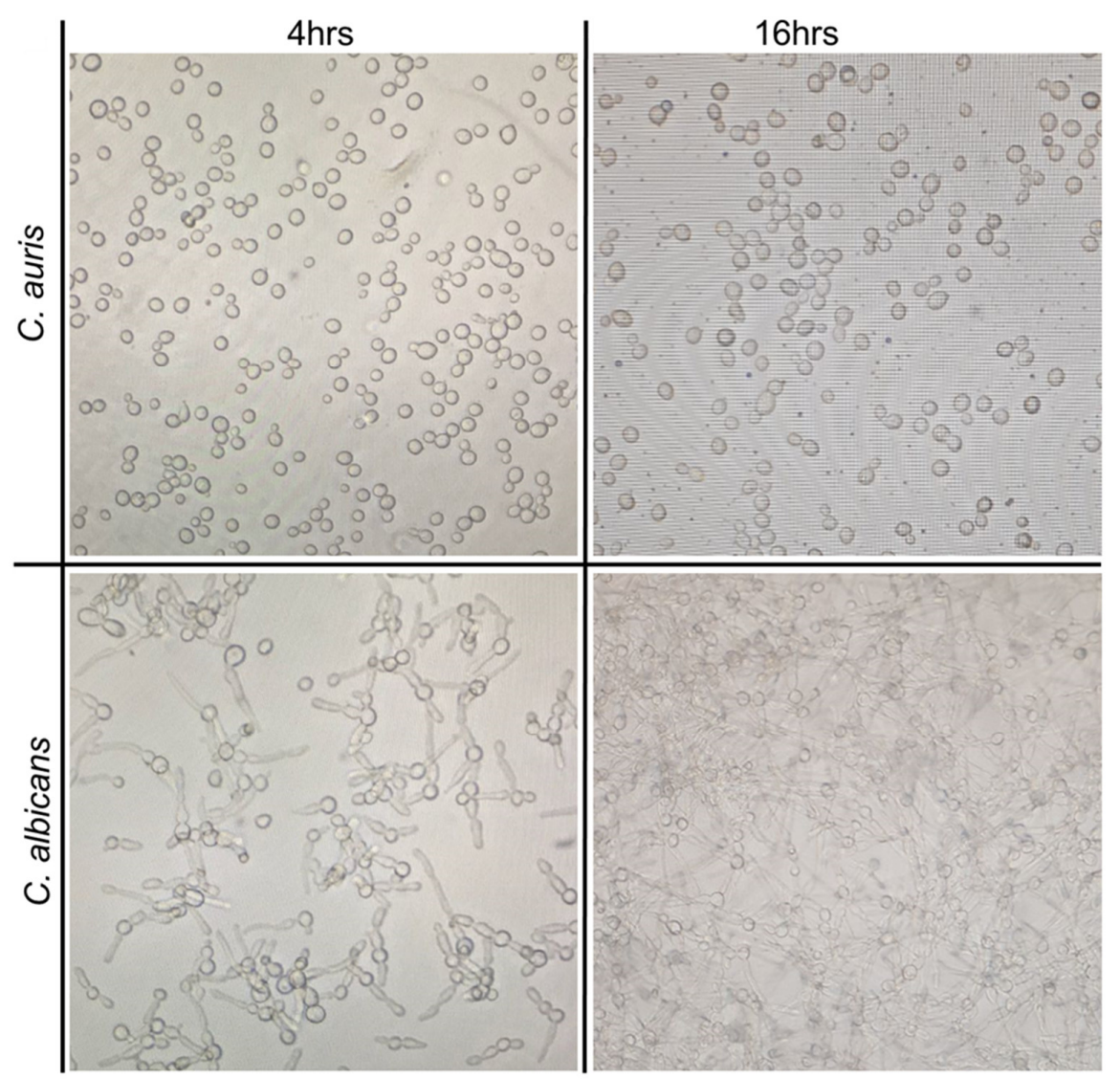

Figure 1. Growth of C. auris (CAU09) in comparison to C. albicans at biofilm-forming condition at 4 and $16 \mathrm{~h}$. Candida isolates were cultured at $37^{\circ} \mathrm{C}$ in $125 \mathrm{~mL}$ Corning culture flasks.

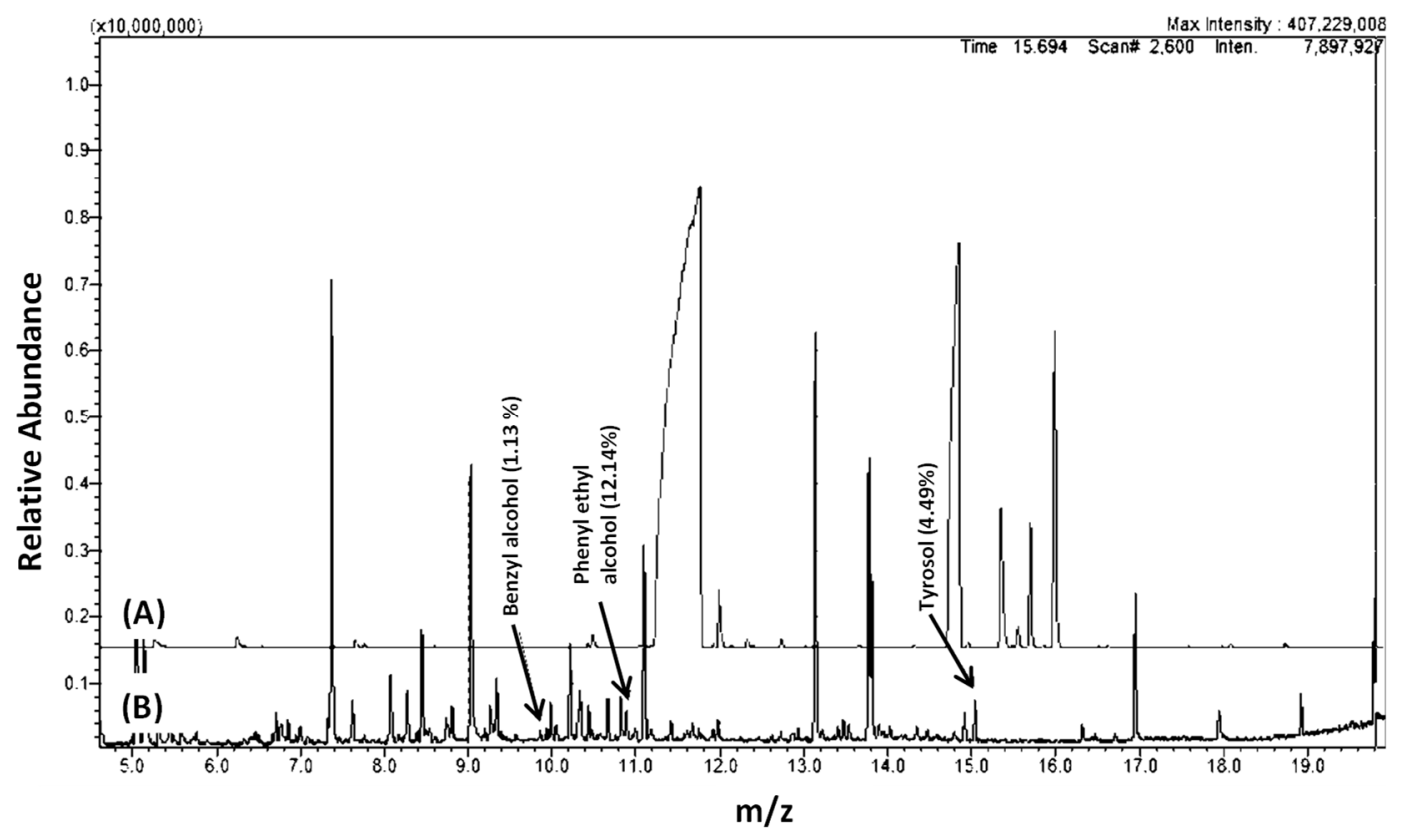

Figure 2. Cont. 


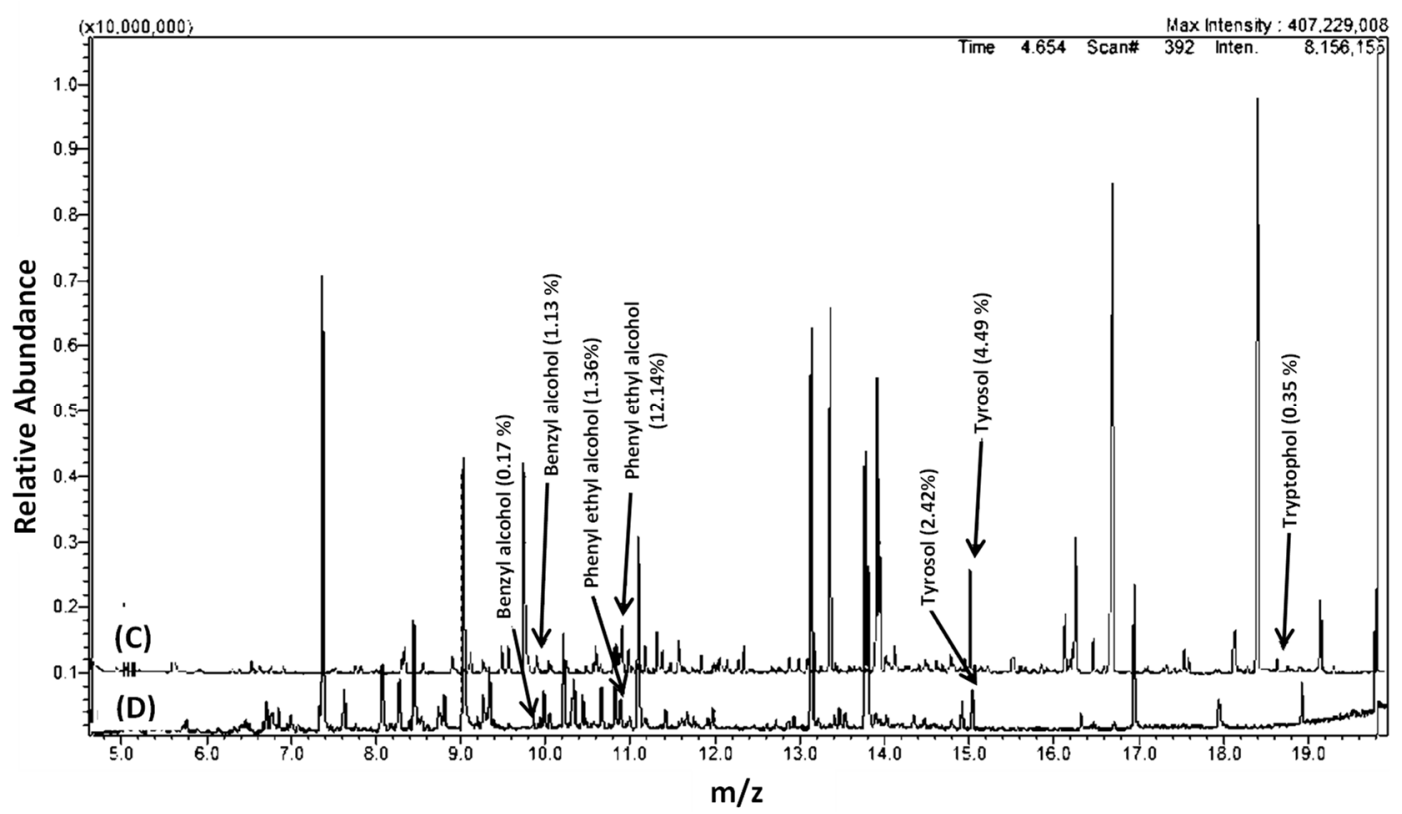

Figure 2. GC-MS chromatograms of Candida cultures extracts. Chromatograms of (A) C. albicans ATCC10231 strain compared to (B) C. auris (CAU09) strain. (C) and (D) GC-MS chromatograms of $C$. auris CAU09 at two different growth stages (C) $16 \mathrm{~h}$ compared to (D) $4 \mathrm{~h}$ incubation periods representing the differences in metabolites abundance. CAU09 was used as a representative to C. auris strains since all C. auris strains showed a similar pattern of GC-MS analysis (data not shown). Cell-free supernatants were collected, extracted by chloroform and derivatized by MSTFA and TMS prior to GC-MS analysis using GC-2010 coupled with a GC-MS QP-2010 Ultra.

The secreted metabolites in CAU09 cultures were further compared at 4 and $16 \mathrm{~h}$ incubation periods (Figure 2C,D). Both phenylethyl alcohol, and tyrosol, were increased by 10 and 4-fold, respectively, within $16 \mathrm{~h}$ of incubation versus $4 \mathrm{~h}$ cultures (Figure 2C,D), while little changes were detected in the levels of benzyl and isoamyl alcohols. It is reported that the aforementioned alcohols including phenylethyl, benzyl and isoamyl alcohols are hyphae-inhibiting metabolites $[3,12,23]$, while tyrosol is a filamentation and biofilm-forming metabolite [16]. The production of phenylethyl, benzyl and isoamyl alcohols is consistent with the yeast form of $C$. auris.

The aforementioned changes in metabolites were compared among four other $C$. auris strains along with C. albicans ATCC10231 (Heatmap in Figure 3, Figure 5A and Supplementary Table S1). A similar pattern was obtained in all other $C$. auris strains. Specifically, C. auris strains secreted high percentages of aromatic alcohols such as phenylethyl alcohol ( 7-11\%), benzyl alcohol $(\sim 0.1-0.2 \%)$, and at a lower extent, isoamyl alcohol. Palmitelaidic acid $(\sim 1-2 \%)$ was also detected in C. auris strains. In contrast, none of these metabolites was identified in C. albicans ATCC10231 except decanoic acid which was detected in very low amount $(0.09 \pm 0.023)$ (Figure 5A and Supplementary Table S1). These results indicate that both phenylethyl alcohol and tyrosol and to some extent palmitelaidic acid are likely critical C. auris morphogenic metabolites at the tested condition. 


\begin{tabular}{|c|c|c|c|c|c|c|}
\hline Metabolites & CAU01 & CAU03 & CAU05 & CAU07 & CAUO9 & C. albicans \\
\hline Benzyl alcohol & 0.171 & 0.094 & 0.18 & 0.19 & 0.21 & 0 \\
\hline Phenylethyl Alcohol & 9.8 & 9.59 & 7.097 & 10.34 & 11.4 & 0 \\
\hline Allyl alcohol & 0 & 0.11 & 0 & 0 & 0 & 0 \\
\hline Isoamyl alcohol & 0.057 & 0.068 & 0.044 & 0.097 & 0.57 & 0 \\
\hline 4-Hydroxybenzyl alcohol & 0 & 0.11 & 0 & 0 & 0 & 0 \\
\hline Diacetone alcohol & 0 & 0 & 0 & 0 & 0 & 0.14 \\
\hline 3,4-Dimethylbenzyl alcohol & 0 & 0 & 0 & 0 & 0 & 0.09 \\
\hline Tyrosol & 4.245 & 3.275 & 3.775 & 4.425 & 3.138 & 0 \\
\hline Decanoic acid (Capric Acid) & 0.754 & 0.268 & 0.248 & 0.403 & 0.502 & 0.09 \\
\hline 10-Undecenoic acid (undecylenic acid) & 0.028 & 0.034 & 0.047 & 0.055 & 0.345 & 0 \\
\hline Palmitelaidic acid & 0.14 & 0.51 & 1.84 & 0.04 & 0.75 & 0 \\
\hline Hexanoic acid (caproic acid) & 0.015 & 0.054 & 0.082 & 0.075 & 0.123 & 0 \\
\hline Niacin & 1.46 & 0.16 & 0.29 & 0.37 & 0.37 & 0 \\
\hline Pyrazine derivative & 2.26 & 0.005 & 0.064 & 2.507 & 2.743 & 0 \\
\hline Palmitelaidic acid & 1.02 & 0.939 & 2.072 & 1.133 & 1.815 & 0 \\
\hline Benzeneethanamine & 5.8 & 23.59 & 20.57 & 11.65 & 3.76 & 0 \\
\hline Farnesol & 0 & 0 & 0 & 0 & 0 & 0 \\
\hline 2-Propenoic acid (Acrylic acid) & 0.43 & 0.08 & 0.39 & 0.19 & 0.3 & 0 \\
\hline 3-hydroxy propanoic acid (Hydracrylic acid) & 0.29 & 0.18 & 0.28 & 0.55 & 0.33 & 0 \\
\hline Methionol (3-(Methylthio)propyl alcohol) & 0.099 & 0.082 & 0.19 & 0.918 & 0.865 & 0 \\
\hline Propanoic acid & 0.025 & 0.066 & 0.226 & 0.24 & 0.27 & 11.35 \\
\hline Tryptophol & 0.32 & 0.14 & 0.19 & 0.452 & 0.55 & 0 \\
\hline Benzoic Acid & 0.627 & 0.252 & 0.543 & 1.817 & 0.977 & 0 \\
\hline Octanoic acid (Caprylic acid) & 0.12 & 0.06 & 0.059 & 0.14 & 0.117 & 0 \\
\hline Benzeneacetic acid (phenyl acetic acid) & 1.832 & 0.623 & 1 & 1.57 & 2.37 & 0 \\
\hline Glyceric acid & 1.775 & 0.898 & 0.773 & 0.92 & 0.988 & 0 \\
\hline Kojic acid & 2.24 & 0.407 & 0.5 & 1.668 & 1.79 & 0 \\
\hline Dodecanoic acid (lauric acid) & 0.57 & 0.199 & 0.157 & 0.375 & 0.18 & 0 \\
\hline Pyrrolo[1,2-a]pyrazine-1,4-dione, hexahydro-3-(2-methylpropyl) & 1.32 & 7.74 & 0.74 & 4.6 & 2.27 & 0 \\
\hline 2-Methylpropanoic acid & 0 & 0.41 & 1.47 & 1.43 & 2.99 & 0.09 \\
\hline 4-Methylvaleric acid & 0 & 0.06 & 0 & 0.14 & 0 & 0 \\
\hline Propane, 2-methyl-1,2-bis(trimethylsiloxy) & 0 & 0 & 0.05 & 0.1 & 0 & 0 \\
\hline 2-Pyrrolidinone, TMS derivative & 0.37 & 0 & 0.22 & 0.38 & 0 & 0 \\
\hline (R)-3-Hydroxybutyric acid & 0.12 & 0.07 & 0.24 & 0.34 & 0 & 0 \\
\hline 2-Hydroxy-3-methylbutyric acid & 0 & 0.16 & 0.31 & 0.37 & 0 & 0 \\
\hline 3-Methyl-1,2-bis(trimethylsilyl)butane & 0 & 0 & 0.11 & 0.12 & 0 & 0 \\
\hline 2-Ethoxyethanol & 0.15 & 0 & 0 & 0.45 & 0 & 0 \\
\hline 3,4-Dihydroxy-5-methyl-dihydrofuran-2-one, (D) & 0.15 & 0 & 0 & 0.43 & 0 & 0 \\
\hline Monoethyl succinate & 0 & 0 & 0 & 0.21 & 0 & 0 \\
\hline Thiodiglycol & 0 & 0 & 0 & 0.18 & 0 & 0 \\
\hline Methylsuccinic acid & 0.14 & 0.07 & 0.28 & 0.33 & 0 & 0.27 \\
\hline 2-Hydroxyoctanoic acid & 0.42 & 0.12 & 0.19 & 0.2 & 0 & 0 \\
\hline Pentanedioic acid & 0 & 0.05 & 0.27 & 0.28 & 0 & 0.16 \\
\hline 3-Bromo-1-propanol & 0 & 0 & 0 & 0.88 & 0 & 0 \\
\hline 4-Hydroxy-5-(hydroxymethyl)oxolan-2-one & 0.23 & 0 & 0 & 0.26 & 0 & 0 \\
\hline 3-Phenyllactic acid & 0.23 & 0.07 & 0.19 & 0.2 & 0 & 0 \\
\hline 3-Hydroxyphenylacetic acid & 0.29 & 0.07 & 0.12 & 0.24 & 0 & 0 \\
\hline 3-Octanol, 2-methyl- & 1.12 & 0 & 0.24 & 0.37 & 0 & 0 \\
\hline n-Heptadecanol-1 & 0 & 0 & 0 & 0.55 & 0 & 0 \\
\hline Acedoben & 0 & 0 & 0 & 0.28 & 0 & 0 \\
\hline Indole-5-carboxylic acid & 0 & 0 & 0 & 0.14 & 0 & 0 \\
\hline Carbonic acid, propargyl 2-ethylhexyl ester & 0 & 0 & 0 & 0.11 & 0 & 0.19 \\
\hline Stearic acid & 6.02 & 1.26 & 1 & 0.38 & 0 & 0.17 \\
\hline [1-(3,3-Dimethyloxiran-2-ylmethyl)-3,7-dimethylocta-2,6-dienyl] & 0 & 0 & 0 & 2.39 & 0 & 0 \\
\hline Methylamine & 5.02 & 0 & 4.32 & 1.12 & 3.73 & 0.49 \\
\hline Ethylene glycol & 1.52 & 0.05 & 0.94 & 0.39 & 1.19 & 0.22 \\
\hline 3-Methylbutanoic acid & 0.34 & 0.5 & 1.34 & 2.92 & 3.93 & 0.5 \\
\hline 2-Acetoxyacetate & 0.7 & 0.2 & 0.49 & 0.93 & 1 & 0.38 \\
\hline Lactic Acid & 0.53 & 0.32 & 0.88 & 1.29 & 1.02 & 0.11 \\
\hline Glycolic acid & 0.37 & 0.22 & 0.27 & 0.61 & 0.45 & 0.04 \\
\hline Glycerol & 1.21 & 0.47 & 3.85 & 3.53 & 2.07 & 0.01 \\
\hline Butanedioic acid & 1.12 & 0.79 & 8.81 & 5.16 & 2.82 & 1.99 \\
\hline Myristic acid & 0.5 & 0.15 & 0.7 & 0 & 0.72 & 0.15 \\
\hline Palmitic Acid & 6.64 & 1.85 & 4.65 & 0.63 & 0.91 & 0.57 \\
\hline 4-Isopropylphenol & 0 & 0 & 0 & 0 & 0 & 13.25 \\
\hline Benzene, (1-methylethyl) & 0 & 0 & 0 & 0 & 0 & 5.9 \\
\hline Ethanone, 1-[4-(1-methylethyl)phenyl] & 0 & 0 & 0 & 0 & 0 & 2.24 \\
\hline Butanedioic acid & 1.12 & 0.79 & 8.81 & 5.16 & 2.82 & 0.44 \\
\hline 1-Butanone, 2-chloro-3-methyl-1-[4-(1-methylethyl)phenyl] & 0 & 0 & 0 & 0 & 0 & 0.27 \\
\hline 2-Methyl-6-tert-butylphenol & 0 & 0 & 0 & 0 & 0 & 1.19 \\
\hline
\end{tabular}

Lowest value Highest value

Figure 3. Heatmap of major identified metabolites in Candida cultures by GC-MS. Metabolite average relative percentage of four experimental replicates from two independent biological experiments were displayed as colors ranging from red to green as shown in the key. The relative percentage of a metabolite represented in relation to total areas of all detected metabolites in an extract. 
A)

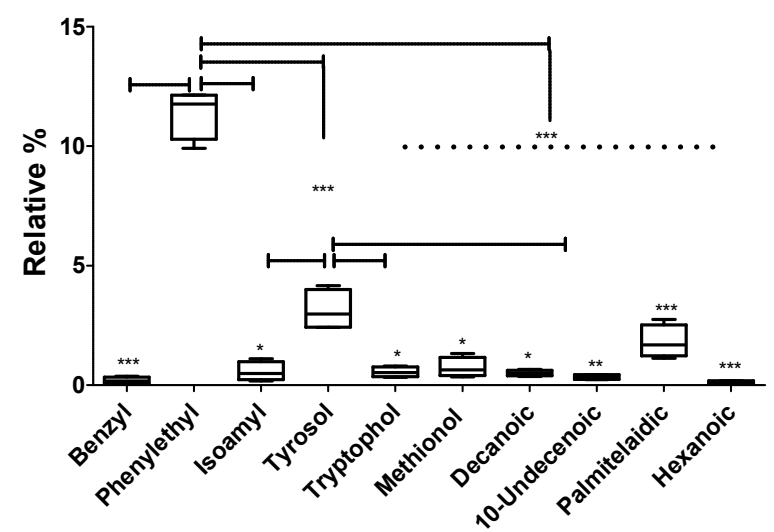

C. auris metabolites
B)

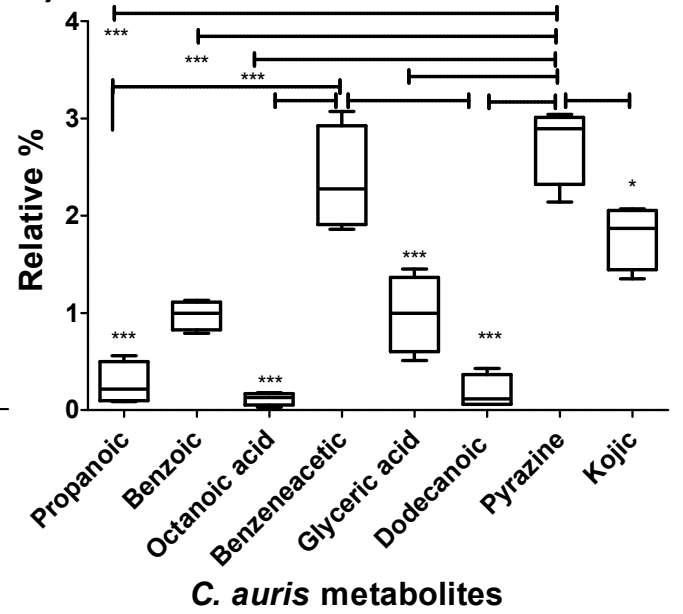

Figure 4. Relative percentages of metabolites produced by C. auris CAU09 cultures. (A) The metabolites produced by the organism for the purpose of its morphogenic changes. (B) The metabolites produced by the organism for the purpose of protection and colonization. The quantity of each metabolite was represented as the relative percentage by measuring the area under the peak of each metabolite in relation to the total areas of all other metabolites detected in the extract. The data display the mean of the relative percentage \pm standard error of the mean. The data was graphed using Box-and-Whiskers Plots and analyzed by one-way analysis of variance (ANOVA) using Bonferroni's Multiple Comparison Test. $P$ value $<0.05$ was considered as significant. The standard error represents the mean of 4 replicas of two independent experiments. The level of significance was indicated by asterisks.

Farnesol was absent in both C. auris and C. albicans cultures. The absence of farnesol in C. albicans culture is expected, since it is a yeast-to-hyphal inhibitory metabolite [15], and the growth condition favored hyphal growth. It is prudent to mention that $C$. auris harbors a homologue for $C$. albicans farnesyl synthase, the rate-limiting enzyme in farnesol biosynthesis [26], with 79\% amino acid identity. Equally important, non-albicans species of Candida are known to produce $\sim 8-35$ times lower levels of farnesol when compared to C. albicans [27]. Therefore, the lack of detection of any farnesol could be due to lower limits of production consistent with what is seen with non-albicans species. This result indicated that farnesol expression was not required in C. auris culture condition under study and hence confirmed that other factors may be involved in maintaining the growth of $C$. auris as yeast form. Importantly, farnesol is known to have reversal effect on tyrosol [16]; and production of tyrosol by C. auris was favored at this condition, a condition suitable for biofilm development. Collectively, these results indicate that fundamental biological processes are under complex positive and negative control by environmental conditions in which these aromatic alcohols are secreted. Future studies focusing on gene expression analysis and investigation of transcription factors that govern yeast-to-hyphae switch might shed light on the reasons why $C$. auris lacks the ability to form true hyphae.

Decanoic, 10-undecenoic and palmitelaidic were acid metabolites identified in C. auris cultures that have never been detected by other Candida Spp. (Figure 3, Figure 5A and Supplementary Table S1). This result raises the possibility of using these acid metabolites as biomarkers to aid in the organism diagnosis. The detected metabolites were also reported as hyphae-inhibitory substances [28-30], a situation may favor the growth of the organism in the yeast form. 


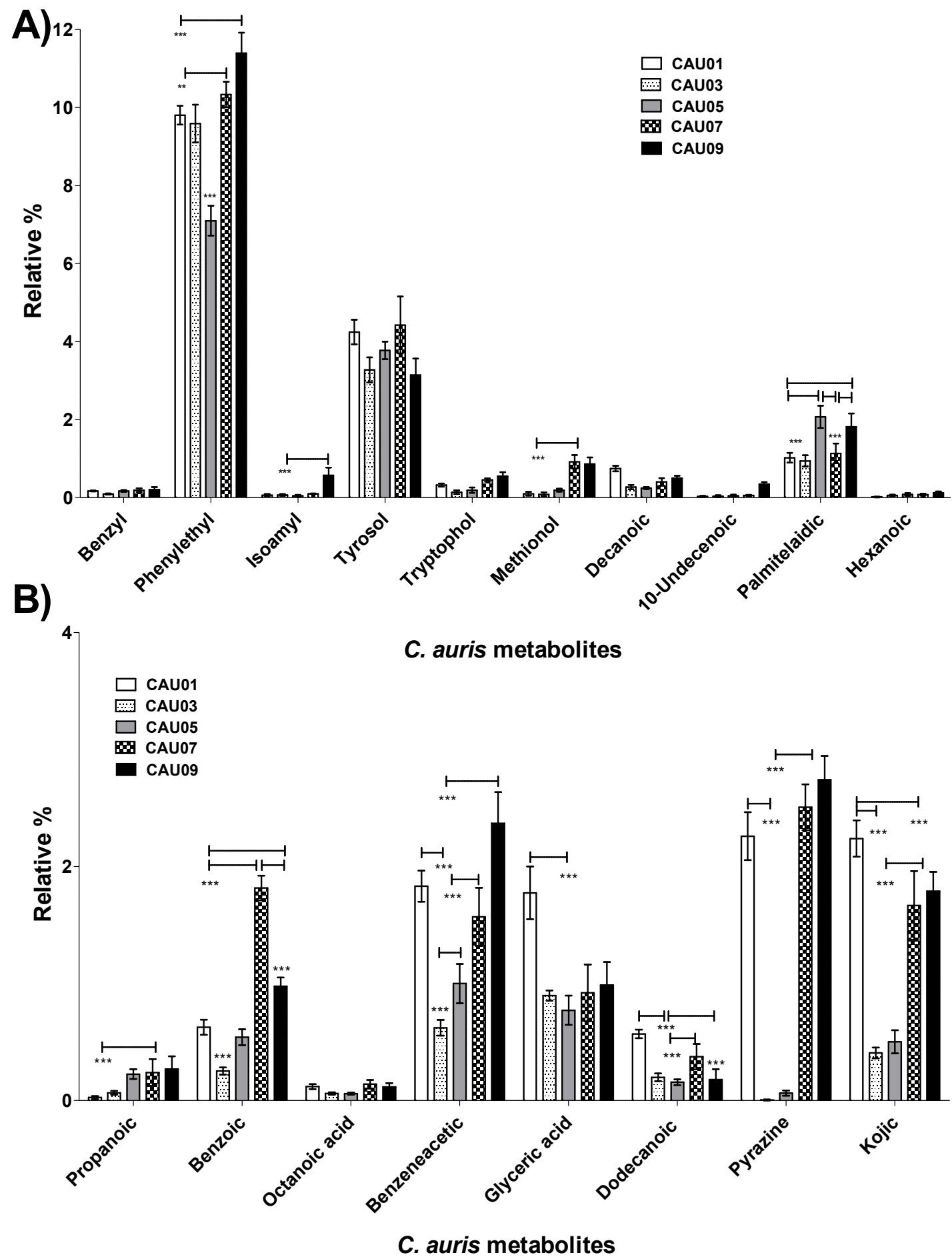

Figure 5. Comparison of relative percentages of metabolites produced by different C. auris strains. (A) Proposed metabolites employed in morphogenic changes. (B) Proposed metabolites produced by the organism for the purpose of protection and colonization. The quantity of each metabolite was represented as the relative percentage by measuring the area under the peak of each metabolite in relation to the total areas of all other metabolites detected in the extract. The data display the mean of the relative percentage \pm standard error of the mean. The data was analyzed by two-way analysis of variance (ANOVA). $P$ value $<0.05$ was considered as significant. The standard error represents the mean of 4 experimental replicates of two independent biological experiments. The level of significance was indicated by asterisks. 


\subsection{C. auris Produced Auto-Protective/Auto-Toxic Metabolites}

Some microbial metabolites exert toxic effects on host cells and/or modulate host immunity in order to facilitate microbial invasion [31]. C. auris secreted fatty acids such as capric, undecylenic and palmitelaidic acids (Figures 3,4A and 5A), known to impair the ability of host natural response to eliminate microorganisms [32]. C. auris also secreted propanoic acid that can exert immune-suppressive activity [33] and pyrazine derivative that may help in host-microbe colonization [34] (Figures 4B and 5B). Furthermore, C. auris produced autotoxic metabolites including tryptophol [35], propanoic [36], benzoic [37], octanoic acid (caprylic acid) [38], benzeneacetic acid (phenyl acetic acid) [37], glyceric [39], kojic [40,41], dodecanoic (lauric) [42,43] acids and pyrrolo[1,2-a]pyrazine-1,4-dione, hexahydro-3-(2-methylpropyl) [44] that can facilitate its own growth and hence access more nutrients (Figures 4B and 5B). Although the production of the aforementioned metabolites was differ between different $C$. auris strains, pyrazine derivative, benzeneacetic acid, kojic acid, glyceric acid and benzoic acid were produced in higher levels when compared to other auto-protective metabolites (Figures $4 \mathrm{~B}$ and $5 \mathrm{~B}$ ), which may indicate their favored production by the organism. On the other hand, out of the aforementioned auto-protective metabolites, C. albicans showed production of very high level of propanoic acid ( 11.35 \pm 0.24) (Figure 3 and Supplementary Table S1). Autotoxic/auto-protective metabolites are known to increase nutrient availability and hence allow better colonization of the pathogenic organism in addition to its immune-suppressive effects [35].

\subsection{C. auris Produced Metabolic Fermentation Products, Known for Colonization and Invasion}

Within the host, the pathogen must efficiently compete for nutrients with host cells [45] and its metabolism is affected in a niche-specific fashion [46]. Microbial pathogens produce metabolic products to overcome the host resistance mechanisms and hence allow the pathogen to colonize and invade. Such metabolic fermentation products may modulate the host immune system and change the body temperature or $\mathrm{pH}[47,48]$. Compared to C. albicans, C. auris produced fermentation metabolic products including hexanoic acid (caproic acid), 2,3-butanediol [49], 2-propenoic acid (acrylic acid) [50], 3-hydroxypropanoic acid (hydracrylic acid) [51] and the yeast-specific fermentation product methionol [52] (Figure 3 and Supplementary Table S1).

\section{Materials and Methods}

\subsection{Organisms and Culture Conditions}

Five different $C$. auris clinical isolates were obtained from Centers for Disease Control and Prevention (CDC, Atlanta, GA, USA). These were CAU-01 (East Asian clade, ear), CAU-03 (African clade, blood), CAU-05 (South American clade, blood), CAU-07 (South Asian clade, blood), and CAU-09 (South Asian clade, bronchoalveolar lavage [BAL]). C. albicans reference strain ATCC10231 was used as a control. Candida isolates were allowed to grow separately in yeast nitrogen base (YNP) supplemented with $2 \%$ glucose at $37{ }^{\circ} \mathrm{C}$ for $16 \mathrm{~h}$. Cells were washed with phosphate buffered saline (PBS), and the inoculums adjusted to $5 \times 10^{6}$ yeast/mL with RPMI-1640 (Sigma-Aldrich, Torrance, CA, USA) using hemacytometer [53]. The cultures were further incubated for $24 \mathrm{~h}$ at $37^{\circ} \mathrm{C}$ in $125 \mathrm{~mL} \mathrm{Corning}$ culture flasks, after which cell-free supernatants were collected by filtration using $0.2 \mu \mathrm{M}$ Whatman filter paper.

\subsection{Preparation of Samples for GC-MS Analysis}

The filtered supernatants collected separately from each Candida isolate was extracted with chloroform (Fisher Scientific, Santa Clara, CA, USA). The chloroform layer was dehydrated over anhydrous sodium sulphate (Fisher Scientific) followed by evaporation using rotatory evaporator (Buchi, Essen, Germany). The residue collected from each extracted culture was dissolved in $500 \mu \mathrm{L}$ chloroform prior to GC-MS injection. Furthermore, $100 \mu \mathrm{L}$ of the chloroform extract was derivatized 
by adding $50 \mu \mathrm{L}$ of $N$-trimethylsilyl- $N$-methyl trifluoroacetamide and trimethylchlorosilane (MSTFA + $1 \%$ TMS) followed by incubation at $50{ }^{\circ} \mathrm{C}$ for $30 \mathrm{~min}$ prior to GC-MS analysis.

\subsection{GC-MS Spectrometry}

GC-MS analysis was performed using a QP2010 gas chromatography-mass spectrometer (GC-2010 coupled with a GC-MS QP-2010 Ultra) equipped with an auto-sampler (AOC-20i+s) from Shimadzu (Tokyo, Japan), using Rtx-5ms column ( $30 \mathrm{~m}$ length $\times 0.25 \mathrm{~mm}$ inner diameter $\times 0.25 \mu \mathrm{m}$ film thickness; Restek, Bellefonte, PA, USA). Helium (99.9\% purity) was used as the carrier gas with the column flow rate of $1 \mathrm{~mL} / \mathrm{min}$. The column temperature regime was initially adjusted at $35^{\circ} \mathrm{C}$ for $2 \mathrm{~min}$; followed by an increase in a rate of $10^{\circ} \mathrm{C} / \mathrm{min}$ to reach $250^{\circ} \mathrm{C}$. The temperature was then increased by $20^{\circ} \mathrm{C} / \mathrm{min}$ until reaching $320^{\circ} \mathrm{C}$ and kept for $23 \mathrm{~min}$. The injection volume and injection temperature were $1 \mu \mathrm{L}$ and $250^{\circ} \mathrm{C}$ using splitless injection mode, respectively. The mass spectrometer operated in electron compact mode with electron energy of $70 \mathrm{eV}$. Both the ion source temperature and the interface temperature were set at $240{ }^{\circ} \mathrm{C}$ and $250{ }^{\circ} \mathrm{C}$, respectively. The MS mode was set on scan mode starting from 35 to $450 \mathrm{~m} / z$ with a scan speed of 1428 . Data collection and analysis were performed using MSD Enhanced Chemstation software (Shimadzu). Product spectra were identified by comparison of the measured fragmentation patterns to those found in the NIST 08 Mass Spectral Library.

\subsection{Bioinformatics detection of farnesyl synthase protein in C. auris}

A local BLAST database of $C$. auris genome was created using Basic Local Alignment Search Tool plus (BLAST+) [54]. Farnesyl synthase protein sequence (Accession \#KGQ89011) from C. albicans was downloaded from NCBI and used to search the generated $C$. auris genome using tblastn command. The search revealed a sequence with $79 \%$ identity within 577 score.

\subsection{Statistical analysis}

The data was collected and graphed using either Excel to generate the heatmap or Graph Pad (5.04, La Jolla, CA, USA) for Windows to generate the Box-and-Whiskers Plots and group comparison. The quantification of metabolites extracts were analyzed by one-way analysis of variance (ANOVA) using Bonferroni's Multiple Comparison Test or two-way analysis of variance as indicated per each graph. $P$ value $<0.05$ was considered significant. The standard error represents the mean of 4 replicas of two independent experiments (two experimental replicates per each independent biological experiment). Each extract was divided into two and extracted separately followed by derivatization prior to GC-MS analysis.

\section{Conclusions}

Metabolites produced by microorganisms may be used as communication signals that allow the microorganisms to share information and hence provide some regulatory responses during infection. Metabolic profiling can be of great help to identify critical determinant of pathogens and hence can control disease progression. Candida spp. is known for secreting metabolites that control its morphogenesis. C. auris is a newly-emergent Candida species that is maintained in the yeast phenotype during its growing stages. Analysis of $C$. auris cultures by GC-MS showed that the fungus produces diverse hyphae-inhibiting metabolites in addition to biofilm-forming tyrosol that are distinct from $C$. albicans. The results provided in this research is the first to identify C. auris metabolic profiling; and thus can shed light on the virulence of this multi-drug resistant yeast and may eventually lead to the development of new strategies for in deep investigation including gene expression analysis and study of morphogenesis-regulatory pathways.

Supplementary Materials: The supplementary materials are available online.

Author Contributions: Conceptualization, S.S.M.S.; methodology, S.S.M.S., M.H.S., B.Q.S. and A.A.; software, S.S.M.S.; validation, S.S.M.S., A.S.I. and M.H.S.; formal analysis, S.S.M.S. and A.S.I.; investigation, S.S.M.S. and 
A.S.I.; resources, S.S.M.S., M.H.S., B.Q.S. and A.S.I.; data curation, S.S.M.S.; writing-original draft preparation, S.S.M.S., M.H.S., B.Q.S., P.U. and A.S.I.; writing-review and editing, S.S.M.S. and A.S.I.; visualization, S.S.M.S.; supervision, S.S.M.S.; project administration, S.S.M.S. and A.S.I.; funding acquisition, S.S.M.S.

Funding: This research received no external funding.

Acknowledgments: This work was supported by grants from University of Sharjah, U.A.E. to S.S.M.S.

Conflicts of Interest: The authors declare no conflict of interest.

\section{References}

1. Wisplinghoff, H.; Bischoff, T.; Tallent, S.M.; Seifert, H.; Wenzel, R.P.; Edmond, M.B. Nosocomial bloodstream infections in us hospitals: Analysis of 24,179 cases from a prospective nationwide surveillance study. Clin. Infect. Dis. 2004, 39, 309-317. [CrossRef]

2. Arendrup, M.C.; Prakash, A.; Meletiadis, J.; Sharma, C.; Chowdhary, A. Comparison of EUCAST and CLSI reference microdilution MICs of eight antifungal compounds for Candida auris and associated tentative epidemiological cutoff values. Antimicrob. Agents Chemoth. 2017, 61, e00485-17. [CrossRef] [PubMed]

3. Kathuria, S.; Singh, P.K.; Sharma, C.; Prakash, A.; Masih, A.; Kumar, A.; Meis, J.F.; Chowdhary, A. Multidrug-resistant Candida auris misidentified as Candida haemulonii: Characterization by matrix-assisted laser desorption ionization-time of flight mass spectrometry and DNA sequencing and its antifungal susceptibility profile variability by vitek 2, clsi broth microdilution, and etest method. J. Clin. Microbiol. 2015, $53,1823-1830$.

4. CDC. Candida auris Interim Recommendations for Healthcare Facilities and Laboratories |Fungal Diseases I CDC. Available online: https:www.cdc.gov/fungal/diseases/candidiasis/recommendations.html (accessed on 21 September 2018).

5. Al-Siyabi, T.; Al Busaidi, I.; Balkhair, A.; Al-Muharrmi, Z.; Al-Salti, M.; Al'Adawi, B. First report of Candida auris in Oman: Clinical and microbiological description of five candidemia cases. J. Infect. 2017, 75, 373-376. [CrossRef] [PubMed]

6. Satoh, K.; Makimura, K.; Hasumi, Y.; Nishiyama, Y.; Uchida, K.; Yamaguchi, H. Candida auris sp. nov., a novel ascomycetous yeast isolated from the external ear canal of an inpatient in a Japanese hospital. Microbiol. Immun. 2009, 53, 41-44. [CrossRef]

7. Sears, D.; Schwartz, B.S. Candida auris: An emerging multidrug-resistant pathogen. Int. J. Infect. Dis. 2017, 63, 95-98. [CrossRef]

8. Lockhart, S.R.; Etienne, K.A.; Vallabhaneni, S.; Farooqi, J.; Chowdhary, A.; Govender, N.P.; Colombo, A.L.; Calvo, B.; Cuomo, C.A.; Desjardins, C.A.; et al. Simultaneous emergence of multidrug-resistant Candida auris on 3 continents confirmed by whole-genome sequencing and epidemiological analyses. Clin. Infect. Dis. 2017, 64, 134-140. [CrossRef] [PubMed]

9. Rudramurthy, S.M.; Chakrabarti, A.; Paul, R.A.; Sood, P.; Kaur, H.; Capoor, M.R.; Kindo, A.J.; Marak, R.S.K.; Arora, A.; Sardana, R.; et al. Candida auris candidaemia in Indian ICUs: Analysis of risk factors. J. Antimicrob. Chemother. 2017, 72, 1794-1801. [CrossRef]

10. Chowdhary, A.; Sharma, C.; Meis, J.F. Candida auris: A rapidly emerging cause of hospital-acquired multidrug-resistant fungal infections globally. PLOS Pathog. 2017, 13, e1006290. [CrossRef]

11. Choi, H.I.; An, J.; Hwang, J.J.; Moon, S.-Y.; Son, J.S. Otomastoiditis caused by Candida auris: Case report and literature review. Mycoses 2017, 60, 488-492. [CrossRef]

12. Wang, X.; Bing, J.; Zheng, Q.; Zhang, F.; Liu, J.; Yue, H.; Tao, L.; Du, H.; Wang, Y.; Wang, H.; et al. The first isolate of Candida auris in China: Clinical and biological aspects. Emerg. Microbiol. Infect. 2018, 7, 93. [CrossRef]

13. Miller, M.B.; Bassler, B.L. Quorum Sensing in Bacteria. Annu. Rev. Microbiol. 2001, 55, 165-199. [CrossRef]

14. Williams, P. Quorum sensing, communication and cross-kingdom signalling in the bacterial world. Microbiology 2007, 153, 3923-3938. [CrossRef] [PubMed]

15. Oh, K.-B.; Miyazawa, H.; Naito, T.; Matsuoka, H. Purification and characterization of an autoregulatory substance capable of regulating the morphological transition in Candida albicans. Proc. Natl. Acad. Sci. USA 2001, 98, 4664-4668. [CrossRef] [PubMed]

16. Chen, H.; Fujita, M.; Feng, Q.; Clardy, J.; Fink, G.R. Tyrosol is a quorum-sensing molecule in Candida albicans. Proc. Natl. Acad. Sci. USA 2004, 101, 5048-5052. [CrossRef] [PubMed] 
17. Han, T.-L.; Tumanov, S.; Cannon, R.D.; Villas-Boas, S.G. Metabolic response of Candida albicans to phenylethyl alcohol under hyphae-inducing conditions. PLoS ONE 2013, 8, e71364. [CrossRef] [PubMed]

18. Martins, M.; Henriques, M.; Azeredo, J.; Rocha, S.M.; Coimbra, M.A.; Oliveira, R. Morphogenesis control in Candida albicans and Candida dubliniensis through signaling molecules produced by planktonic and biofilm cells. Eukaryot. Cell. 2007, 6, 2429-2436. [CrossRef]

19. Faber, J.H.; Malmodin, D.; Toft, H.; Maher, A.D.; Crockford, D.; Holmes, E.; Nicholson, J.K.; Dumas, M.E.; Baunsgaard, D. Metabonomics in diabetes research. J. Diab. Sci. Technol. 2007, 1, 549-557. [CrossRef]

20. Gow, N.A.R.; Brown, A.J.P.; Odds, F.C. Fungal morphogenesis and host invasion. Curr. Opin. Microbiol. 2002, 5, 366-371. [CrossRef]

21. Sudbery, P.; Gow, N.; Berman, J. The distinct morphogenic states of Candida albicans. Trend Microbiol. 2004, 12, 317-324. [CrossRef]

22. Albuquerque, P.; Casadevall, A. Quorum sensing in fungi-A review. Med. Mycol. 2012, 50, $337-345$. [CrossRef] [PubMed]

23. Chowdhary, A.; Anil Kumar, V.; Sharma, C.; Prakash, A.; Agarwal, K.; Babu, R.; Dinesh, K.R.; Karim, S.; Singh, S.K.; Hagen, F.; et al. Multidrug-resistant endemic clonal strain of Candida auris in India. Europ. J. Clin. Microbiol. Infect. Dis. 2014, 33, 919-926. [CrossRef]

24. Oh, B.J.; Shin, J.H.; Kim, M.-N.; Sung, H.; Lee, K.; Joo, M.Y.; Shin, M.G.; Suh, S.P.; Ryang, D.W. Biofilm formation and genotyping of Candida haemulonii, Candida pseudohaemulonii, and a proposed new species (Candida auris) isolates from Korea. Med. Mycol. 2011, 49, 98-102. [CrossRef] [PubMed]

25. Larkin, E.; Hager, C.; Chandra, J.; Mukherjee, P.K.; Retuerto, M.; Salem, I.; Long, L.; Isham, N.; Kovanda, L.; Borroto-Esoda, K.; et al. The emerging pathogen Candida auris: Growth phenotype, virulence factors, activity of antifungals, and effect of scy-078, a novel glucan synthesis inhibitor, on growth morphology and biofilm formation. Antimicrob. Agents Chemother. 2017, 61, e02396-16. [CrossRef] [PubMed]

26. Hornby, J.M.; Kebaara, B.W.; Nickerson, K.W. Farnesol biosynthesis in Candida albicans: Cellular response to sterol inhibition by zaragozic acid B. Antimicrob. Agents Chemother. 2003, 47, 2366-2369. [CrossRef] [PubMed]

27. Weber, K.; Schulz, B.; Ruhnke, M. The quorum-sensing molecule E,E-farnesol-Its variable secretion and its impact on the growth and metabolism of Candida species. Yeast 2010, 27, 727-739. [CrossRef]

28. Murzyn, A.; Krasowska, A.; Stefanowicz, P.; Dziadkowiec, D.; Łukaszewicz, M. Capric acid secreted by $S$. boulardii inhibits C. albicans filamentous growth, adhesion and biofilm formation. PLoS ONE 2010, 5, e12050. [CrossRef] [PubMed]

29. McLain, N.; Ascanio, R.; Baker, C.; Strohaver, R.A.; Dolan, J.W. Undecylenic acid inhibits morphogenesis of Candida albicans. Antimicrob. Agents Chemother. 2000, 44, 2873-2875. [CrossRef]

30. Shareck, J.; Nantel, A.; Belhumeur, P. Conjugated linoleic acid inhibits hyphal growth in Candida albicans by modulating ras1p cellular levels and downregulating TEC1 expression. Eukaryot. Cell. 2011, 10, 565-577. [CrossRef]

31. Winzer, K.; Williams, P. Quorum sensing and the regulation of virulence gene expression in pathogenic bacteria. Int. J. Med. Microbiol. 2001, 291, 131-143. [CrossRef]

32. De Pablo, M.A.; Ángeles Puertollano, M.; Álvarez De Cienfuegos, G. Immune cell functions, lipids and host natural resistance. FEMS Immunol. Med. Microbiol. 2000, 29, 323-328. [CrossRef]

33. Al-Lahham, S.A.H.; Peppelenbosch, M.P.; Roelofsen, H.; Vonk, R.J.; Venema, K. Biological effects of propionic acid in humans; metabolism, potential applications and underlying mechanisms. Biochimica et Biophysica Acta (BBA)-Mol. Cell. Biol. Lipids 2010, 1801, 1175-1183. [CrossRef]

34. Silva-Junior, E.A.; Ruzzini, A.C.; Paludo, C.R.; Nascimento, F.S.; Currie, C.R.; Clardy, J.; Pupo, M.T. Pyrazines from bacteria and ants: Convergent chemistry within an ecological niche. Sci. Rep. 2018, 8, 2595. [CrossRef] [PubMed]

35. Lingappa, B.T.; Prasad, M.; Lingappa, Y.; Hunt, D.F.; Biemann, K. Phenethyl alcohol and tryptophol: Autoantibiotics produced by the fungus Candida albicans. Science 1969, 163, 192-194. [CrossRef] [PubMed]

36. Yun, J.; Lee, D.G. A novel fungal killing mechanism of propionic acid. FEMS Yeast Res. 2016, 16, fow089. [CrossRef] [PubMed]

37. Teodoro, G.R.; Ellepola, K.; Seneviratne, C.J.; Koga-Ito, C.Y. Potential use of phenolic acids as anti-Candida agents: A review. Front. Microbiol. 2015, 6, 1420. [CrossRef]

38. Tsukahara, T. Fungicidal action of caprylic acid for Candida albicans ii. Possible mechanisms of the action. Jpn. J. Microbiol. 1962, 6, 1-14. [CrossRef] 
39. Cottier, F.; Tan, A.S.M.; Xu, X.; Wang, Y.; Pavelka, N. MIG1 regulates resistance of Candida albicans against the fungistatic effect of weak organic acids. Eukaryot. Cell. 2015, 14, 1054-1061. [CrossRef]

40. Chee, H.Y.; Lee, E.H. Fungistatic activity of kojic acid against human pathogenic fungi and inhibition of melanin-production in Cryptococcus neoformans. Mycobiology 2003, 31, 248-250. [CrossRef]

41. Vijayalakshmi, P.; Durgadevi, K.B. A study on anti-fungal activity of kojic acid synthesized by Aspergillus flavus FJ537130 strain isolated from peanut soil. Asian J. Microbiol. Biotechnol. Environ. Sci. 2017, 19, 160-164.

42. Bergsson, G.; Arnfinnsson, J.; Steingrímsson, Ó.; Thormar, H. In vitro killing of Candida albicans by fatty acids and monoglycerides. Antimicrob. Agents Chemother. 2001, 45, 3209-3212. [CrossRef] [PubMed]

43. Pohl, C.; Kock, L.F.; Thibane, V. Antifungal free fatty acids: A Review. Sci. Microb. Pathog. Curr. Res. Technol. Adv. 2011, 1, 61-71.

44. Sanjenbam, P.; Kannabiran, K. Bioactivity of pyrrolo[1,2-a]pyrazine-1,4-dione,hexahydro-3-(phenylmethyl)extracted from Streptomyces sp. Vitpk9 isolated from the salt spring habitat of Manipur, India. Asian J. Pharm. 2016, 10, 265-270.

45. Bäckhed, F.; Ley, R.E.; Sonnenburg, J.L.; Peterson, D.A.; Gordon, J.I. Host-bacterial mutualism in the human intestine. Science 2005, 307, 1915-1920. [CrossRef]

46. Barelle, C.J.; Priest, C.L.; MacCallum, D.M.; Gow, N.A.R.; Odds, F.C.; Brown, A.J.P. Niche-specific regulation of central metabolic pathways in a fungal pathogen. Cell. Microbiol. 2006, 8, 961-971. [CrossRef] [PubMed]

47. Passalacqua, K.D.; Charbonneau, M.-E.; O’Riordan, M.X.D. Bacterial metabolism shapes the host:pathogen interface. Microbiol. Spectr. 2016, 4. [CrossRef]

48. Van Baarlen, P.; Van Belkum, A.; Summerbell, R.C.; Crous, P.W.; Thomma, B.P.H.J. Molecular mechanisms of pathogenicity: How do pathogenic microorganisms develop cross-kingdom host jumps? FEMS Microbiol. Rev. 2007, 31, 239-277. [CrossRef]

49. Yang, T.; Rao, Z.; Zhang, X.; Xu, M.; Xu, Z.; Yang, S.-T. Metabolic engineering strategies for acetoin and 2,3-butanediol production: Advances and prospects. Crit. Rev. Biotechnol. 2017, 37, 990-1005. [CrossRef]

50. Zhu, L.; Chen, H.; Huang, L.; Cai, J.; Xu, Z. Electrochemical analysis of Clostridium propionicum and its acrylic acid production in microbial fuel cells. Eng. Life Sci. 2011, 11, 238-244. [CrossRef]

51. Maris, A.J.A.V.; Konings, W.N.; Dijken, J.P.V.; Pronk, J.T. Microbial export of lactic and 3-hydroxypropanoic acid: Implications for industrial fermentation processes. Met. Eng. 2004, 6, 245-255. [CrossRef]

52. Seow, Y.-X.; Ong, P.K.C.; Liu, S.-Q. Production of flavour-active methionol from methionine metabolism by yeasts in coconut cream. Int. J. Food Microbiol. 2010, 143, 235-240. [CrossRef] [PubMed]

53. Ibarra-Trujillo, C.; Villar-Vidal, M.; Gaitán-Cepeda, L.A.; Pozos-Guillen, A.; Mendoza-de Elias, R.; Sánchez-Vargas, L.O. Ensayo de formación y cuantificación de biopelículas mixtas de Candida albicans y Staphylococcus aureus. Revista Iberoamericana de Micología 2012, 29, 214-222. [CrossRef] [PubMed]

54. Basic Local Alignment Search Tool (BLAST). Available online: http://blast.ncbi.nlm.nih.gov/Blast.cgi? PAGE_TYPE=BlastDocs\&DOC_TYPE=Download (accessed on 20 November 2018).

(C) 2019 by the authors. Licensee MDPI, Basel, Switzerland. This article is an open access article distributed under the terms and conditions of the Creative Commons Attribution (CC BY) license (http://creativecommons.org/licenses/by/4.0/). 